\title{
Corrigendum
}

\section{Effects of synbiotic supplementation on metabolic parameters and apelin in women with polycystic ovary syndrome: a randomised double-blind placebo-controlled trial - Corrigendum}

Elham Karimi, Ashraf Moini, Mehdi Yaseri, Nooshin Shirzad, Mahdi Sepidarkish, Mojgan Hossein-Boroujerdi and Mohammad Javad Hosseinzadeh-Attar

doi: $10.1017 /$ S0007114517003920

In the published version of this article, there was an error in reporting the final number of the patients who finished the study. In this study, data for 45 patients in the intervention and 43 patients in the placebo group were analyzed and reported based on appropriate statistical tests (t-test or ANCOVA). At baseline, 99 patients were randomized to two groups to receive either synbiotic $(n=50)$ or placebo $(n=49)$. Five patients in the intervention and 6 in the placebo group did not finish the study. This correction should be considered in the Abstract, main text, and CONSORT flow chart.

In Table 1, the range values reported for BMI are in fact "Inter-quartile range" values.

In Table 2, the correct difference and 95\% CI values for post- and change values of fibre intake are as below:
Post- fibre:
Difference: -0.326;
$95 \%$ CI: -0.93 to 0.28

Change- fibre:

Difference: -0.003

95\% CI: -0.38 to 0.37 